\title{
Linear Gravitational Instability of Filamentary and Sheet-like Molecular Clouds with Magnetic Fields
}

\author{
Curtis S. Gehman, Fred C. Adams, and Richard Watkins ${ }^{1}$ \\ Physics Department, University of Michigan \\ Ann Arbor, MI 48109-1120
}

\begin{abstract}
We study the linear evolution of small perturbations in self-gravitating fluid systems with magnetic fields. We consider wave-like perturbations to nonuniform filamentary and sheet-like hydrostatic equilibria in the presence of a uniform parallel magnetic field. Motivated by observations of molecular clouds that suggest substantial nonthermal (turbulent) pressure, we adopt equations of state that are softer than isothermal. We numerically determine the dispersion relation and the form of the perturbations in the regime of instability. The form of the dispersion relation is the same for all equations of state considered, for all magnetic field strengths, and for both geometries examined. We demonstrate the existence of a fastest growing mode for the system and study how its characteristics depend on the amount of turbulence and the strength of the magnetic field. Generally, turbulence tends to increase the rate and the length scale of fragmentation. While tending to slow the fragmentation, the magnetic field has little effect on the fragmentation length scale until reaching some threshold, above which the length scale decreases significantly. Finally, we discuss the implications of these results for star formation in molecular clouds.
\end{abstract}

Subject headings: hydrodynamics, instabilities, ISM:clouds, ISM:structure, magnetic fields, turbulence

\footnotetext{
${ }^{1}$ current institution: Dartmouth College, Department of Physics and Astronomy, Hannover, NH 037553528
} 


\section{Introduction}

It is widely believed that most current galactic star formation occurs in molecular clouds (cf. Shu, Adams, \& Lizano 1987 for a review). Observations of these clouds reveal rich and complex structure (e.g., Myers 1991; Blitz 1993). In particular, filamentary and sheet-like structures are common. These structures exhibit a large degree of substructure of their own (e.g., Schneider \& Elmegreen 1979; de Geus, Bronfman, \& Thaddeus 1990; Houlahan \& Scalo 1992; Wiseman \& Adams 1994; Wiseman 1996). Clumps and cores that provide locations for new stars to form are often found along filaments and sheets. Thus, the study of the evolution of molecular clouds and their substructure is important for understanding the processes involved in present day star formation. The goal of this paper is to further our understanding of molecular cloud substructure by studying gravitational instabilities in the presence of both turbulence and magnetic fields.

Most previous work has focused on the case of wave motions and instabilities in uniform density fluids (from Jeans 1928 to Dewer 1970, Langer 1978, Pudritz 1990). Some previous work on wave motions in isothermal molecular cloud filaments and sheets has been done; this work begins with clouds in hydrostatic equilibrium and hence nonuniform density. Many observations show clumps which appear nearly equally spaced along filaments (e.g., Schneider \& Elmegreen 1979; McBreen et al. 1979; Dutrey et al. 1991); this finding has led to the idea that the clumps may arise from a gravitational instability with a particular length scale. Less frequently, it has been proposed that the clumps may be peaks of density waves which propagate along the filament. In the linear regime, both cases are treated by a linear perturbation analysis. Larson (1985) gives a good review of the early progress in doing this type of analysis. More recent work includes Nagasawa (1987), who performed such calculations for an idealized isothermal filament with an axial magnetic field (see also Miyama, Narita, \& Hayashi 1987 for a discussion of sheets). Others (Nakamura, Hanawa, \& Nakano 1991, 1993; Matsumoto, Nakamura, \& Hanawa 1994) have added further embellishments to this model (e.g., rotation). Until recently, however, these studies have typically neglected the effects of turbulence and non-isothermal equations of state. Gehman et al. (1996; hereafter Paper 1) introduced a turbulent equation of state to the problem, but did not include the effects of a magnetic field. When including a magnetic field, one is generally free to choose the form of the unperturbed magnetic field. The results may depend strongly on the form assumed. In this present work, we consider a general barotropic equation of state of the form $p=P(\rho)$ along with a uniform magnetic field. We concentrate this present discussion on equations of state which are softer than isothermal, since these may be the most relevant for molecular clouds (see $\S 2$ ).

A secondary motivation for this work is to generalize the current theory of star forma- 
tion, which typically begins with spherically symmetric clouds (e.g., Larson 1972; Shu 1977; Terebey, Shu, \& Cassen 1984). Models of star formation built upon these collapse calculations are reasonably successful and predict spectral energy distributions of forming stars that are in agreement with observed protostellar candidates Adams, Lada, \& Shu 1987; Butner et al. 1991; Kenyon, Calvet, \& Hartmann 1993). However, departures from spherical symmetry are expected to occur on larger spatial scales, and the collapse of isothermal self-gravitating sheets (Hartmann et al. 1994) and filaments (Inutsuka \& Miyama 1993; Nakamura, Hanawa,

\& Nakano 1995) has begun to be studied. The calculations of this paper help to determine the length scales of fragmentation of molecular cloud sheets and filaments, and the initial conditions for protostellar collapse in nonspherical molecular cloud structures.

This paper is organized as follows. We present the general mathematical framework and discuss equations of state in $\S 2$. The equilibrium solutions of interest are presented and discussed in $\S 3$. In $\S$ we perform a general linear perturbation analysis, the solutions of which are presented and examined in $\$ 5$. We conclude in $\$ 6$ with a discussion and summary of our results.

\section{General Formulation}

We present here the basic equations used to describe self-gravitating fluids with a magnetic field. Throughout this paper, we assume perfect flux freezing. Also, we discuss an equation of state of particular interest - a non-isothermal barotropic equation of state that heuristically includes the effects of the turbulence that is observed in molecular clouds.

In dimensionless units (see Appendix A), the equations of ideal fluid dynamics with self-gravity and a frozen magnetic field can be written in the form

$$
\begin{gathered}
\frac{\partial \rho}{\partial t}+\nabla \cdot(\rho \mathbf{u})=0 \\
\rho \frac{\partial \mathbf{u}}{\partial t}+\rho(\mathbf{u} \cdot \nabla) \mathbf{u}+\nabla p+\rho \nabla \psi-(\nabla \times \mathbf{B}) \times \mathbf{B}=0 \\
\frac{\partial \mathbf{B}}{\partial t}+\nabla \times(\mathbf{B} \times \mathbf{u})=0, \\
\nabla^{2} \psi=\rho,
\end{gathered}
$$

where $\rho$ is the mass density, $\mathbf{u}$ is the fluid velocity, $p$ is the pressure, $\psi$ is the gravitational potential, and $\mathbf{B}$ is the magnetic field strength. We will assume the pressure to have a barotropic form, i.e.,

$$
p=P(\rho) .
$$


Most previous theoretical studies of molecular clouds have assumed an isothermal equation of state, $p=c_{\mathrm{s}}^{2} \rho$, where $c_{\mathrm{s}}$ is the thermal sound speed. We generalize this equation of state by adding a term which attempts to model the "turbulence" observed in molecular clouds. The equation of state takes the form

$$
p=c_{\mathrm{s}}^{2} \rho+p_{0} \log (\rho / \hat{\rho})
$$

where $p_{0}$ is a constant, which may be determined empirically, and $\hat{\rho}$ is an arbitrary reference density (Lizano \& Shu 1989). In this paper, we will always take our dimensionless density variable $\rho$ to be 1 at the center of equilibrium configurations, so that $\hat{\rho}=\rho_{\mathrm{c}}$, the central density (see Appendix $\mathrm{A}$ ). The logarithmic nature of the turbulence term arises from the empirical linewidth-density relation $\Delta v \propto \rho^{-1 / 2}$ (Larson 1981; Myers 1983; Dame et al. 1986; Myers \& Fuller 1992). After transforming to dimensionless variables, this equation of state can be written as

$$
p=P(\rho)=\rho+\kappa \log \rho,
$$

where the turbulence parameter $\kappa$ is defined by

$$
\kappa=p_{0} / c_{\mathrm{s}}^{2} \hat{\rho}
$$

Typically, we are interested in clouds with number densities of about $1000 \mathrm{~cm}^{-3}$ and thermal sound speed $c_{\mathrm{s}} \sim 0.20 \mathrm{~km} \mathrm{sec}^{-1}$. Observational considerations suggest that $p_{0}$ ranges from 10 to 70 picodyne $/ \mathrm{cm}^{2}$ (Myers \& Goodman 1988; Solomon et al. 1987). Thus, molecular clouds are expected to have values of the turbulence parameter in the range $6<\kappa<50$. In this work, we want to include both the isothermal limit and the pure "logatropic" limit where the turbulence dominates, so we use the expanded range $0 \leq \kappa<\infty$.

In addition to the empirical motivation for using an equation of state that is softer than isothermal, there exists theoretical support as well. It has been shown that when a molecular cloud begins to collapse (on large spatial scales), a wide spectrum of small scale wave motions can be excited (Arons \& Max 1975; see also Elmegreen 1990). Additional energy input and wave excitation can also be produced by outflows from forming stars (Norman \& Silk 1980). In the presence of magnetic fields, these wave motions generally take the form of magnetoacoustic and Alfvén waves. In any case, these small scale wave motions have velocity perturbations which vary with the gas density in rough agreement with the observations described above. These wave motions can be modeled with an effective equation of state which is softer than isothermal (see Fatuzzo \& Adams 1993; McKee \& Zweibel 1995). 


\section{Static Equilibrium}

We take the unperturbed state of the system to be one of hydrostatic equilibrium $(\mathbf{u} \equiv 0)$. Since the unperturbed magnetic field is uniform, it provides no support. Therefore, the unperturbed state is unaffected by the presence of the magnetic field and, hence, is identical to that discussed in Paper 1. The equilibrium density distribution is determined by the equation

$$
\frac{1}{\rho} \nabla^{2} p-\frac{1}{\rho^{2}} \nabla p \cdot \nabla \rho+\rho=0
$$

\subsection{Filaments}

For the filament, we adopt cylindrical coordinates $(r, \phi, z)$ and assume azimuthal symmetry. The equilibrium equation becomes

$$
\frac{d^{2} \rho}{d r^{2}}+\frac{1}{r} \frac{d \rho}{d r}+\left[\frac{P^{\prime \prime}(\rho)}{P^{\prime}(\rho)}-\frac{1}{\rho}\right]\left(\frac{d \rho}{d r}\right)^{2}+\frac{\rho^{2}}{P^{\prime}(\rho)}=0
$$

where the primes denote derivatives with respect to density, e.g. $P^{\prime}(\rho)=\partial p / \partial \rho$. In the isothermal case, $p=P(\rho)=\rho$, the equilibrium solution is well known (Ostriker 1964) and has the simple form

$$
\rho(r)=\left(1+r^{2} / 8\right)^{-2}
$$

For equations of state which include turbulence $(\kappa \neq 0)$, the solutions have been found numerically (see Paper 1).

Paper I demonstrated an important difference in the asymptotic behavior of the equilibria of the isothermal and turbulent cases. At large radii, the equilibrium density profile of the isothermal filament behaves as $r^{-4}$ while that of the turbulent filament falls off only

as $r^{-1}$. This result implies that the mass per unit length of the isothermal filament remains finite, whereas that of the turbulent filament diverges. The mass per unit length of the isothermal filament is given in dimensionless units by $\mu=8 \pi$. In addition to providing an observable test of the significance of turbulence in clouds, these differences have important implications for the mass scales of fragmentation.

\subsection{Sheets}

For the thick sheet (sometimes referred to as a slab or stratified layer), we use cartesian coordinates $(x, y, z)$ and assume translational symmetry in the $y$ - and $z$-directions. The 
equilibrium equation now becomes

$$
\frac{d^{2} \rho}{d x^{2}}+\left[\frac{P^{\prime \prime}(\rho)}{P^{\prime}(\rho)}-\frac{1}{\rho}\right]\left(\frac{d \rho}{d x}\right)^{2}+\frac{\rho^{2}}{P^{\prime}(\rho)}=0 .
$$

Again, the isothermal solution is well known (Spitzer 1942; Shu 1992)

$$
\rho=\operatorname{sech}^{2}(x / \sqrt{2}),
$$

and for turbulent equations of state the solutions have been found numerically (Paper 1). As in the case of filaments, the asymptotic behavior of the isothermal and turbulent sheets differ significantly. Paper 1 showed that the surface density of the isothermal sheet is $2^{3 / 2}$ and that of the turbulent sheet is infinite.

\section{Perturbations}

We now consider the addition of small perturbations to the equilibrium states described in $\S 3$. We denote the equilibrium quantities with a ' 0 ' subscript and the perturbations with a ' 1 ' subscript. After linearization, the fluid equations (11 -4) can be written

$$
\begin{gathered}
\frac{\partial \rho_{1}}{\partial t}+\nabla \cdot\left(\rho_{0} \mathbf{u}_{1}\right)=0 \\
\rho_{0} \frac{\partial \mathbf{u}_{1}}{\partial t}+\nabla p_{1}+\rho_{0} \nabla \psi_{1}+\rho_{1} \nabla \psi_{0}-\mathcal{L}_{1}=0 \\
\frac{\partial \mathbf{B}_{1}}{\partial t}+\nabla \times\left(\mathbf{B}_{0} \times \mathbf{u}_{1}\right)=0 \\
\nabla^{2} \psi_{1}=\rho_{1}
\end{gathered}
$$

where $\mathcal{L}_{1}$ is the first-order mean Lorentz force per unit volume acting on the fluid and is given by

$$
\mathcal{L}_{1}=\left(\mathbf{B}_{0} \cdot \nabla\right) \mathbf{B}_{1}+\left(\mathbf{B}_{1} \cdot \nabla\right) \mathbf{B}_{0}-\nabla\left(\mathbf{B}_{0} \cdot \mathbf{B}_{1}\right) .
$$

Adopting a barotropic equation of state (5), we may write the pressure perturbation as

$$
p_{1}=P^{\prime}\left(\rho_{0}\right) \rho_{1} \text {. }
$$

We assume that the equilibrium magnetic field is axial, i.e.,

$$
\mathbf{B}_{0}=B_{0}(\varpi) \hat{\mathbf{z}}
$$

and seek solutions for the perturbations of the form

$$
\rho_{1}(\mathbf{x}, t)=f(\varpi) \exp (i k z-i \omega t)
$$




$$
\begin{aligned}
& \mathbf{u}_{1}(\mathbf{x}, t)=\mathbf{v}(\varpi) \exp (i k z-i \omega t), \\
& \mathbf{B}_{1}(\mathbf{x}, t)=\mathbf{b}(\varpi) \exp (i k z-i \omega t), \\
& \psi_{1}(\mathbf{x}, t)=\phi(\varpi) \exp (i k z-i \omega t),
\end{aligned}
$$

where $\varpi$ is a generalized coordinate, which we take as our cartesian $x$ in the case of the sheet and our cylindrical $r$ in the case of the filament. We find purely algebraic expressions for $b_{\varpi}, b_{z}$, and $v_{z}$ :

$$
\begin{gathered}
b_{\varpi}=-\frac{k}{\omega} B_{0} v_{\varpi}, \\
b_{z}=\left(1-\frac{k^{2}}{\omega^{2}} P_{0}^{\prime}\right) \frac{B_{0}}{\rho_{0}} f-\frac{k^{2}}{\omega^{2}} B_{0} \phi+\frac{i B_{0}}{\omega}\left(\frac{1}{\rho_{0}} \frac{d \rho_{0}}{d \varpi}-\frac{1}{B_{0}} \frac{d B_{0}}{d \varpi}+\frac{k^{2} B_{0}}{\omega^{2} \rho_{0}} \frac{d B_{0}}{d \varpi}\right) v_{\varpi}, \\
v_{z}=\frac{k}{\omega}\left(\frac{P_{0}^{\prime}}{\rho_{0}} f+\phi-\frac{i B_{0}}{\omega \rho_{0}} \frac{d B_{0}}{d \varpi} v_{\varpi}\right),
\end{gathered}
$$

and eliminate them from the remaining differential equations. Following a convenient change of one variable,

$$
w \equiv i \omega v_{\varpi}
$$

we obtain the following ordinary differential equations:

$$
\begin{gathered}
\rho \mathcal{D} w+\left(\omega^{2}-k^{2} P^{\prime}\right) f-k^{2} \rho \phi+\left(\frac{d \rho}{d \varpi}+\frac{k^{2}}{\omega^{2}} B \frac{d B}{d \varpi}\right) w=0, \\
{\left[P^{\prime}+\frac{B^{2}}{\rho}\left(1-\frac{k^{2}}{\omega^{2}} P^{\prime}\right)\right] \frac{d f}{d \varpi}+A_{1} f+\left(\rho-\frac{k^{2}}{\omega^{2}} B^{2}\right) \frac{d \phi}{d \varpi}+A_{2} \phi+A_{3} w=0,} \\
\mathcal{D} \frac{d \phi}{d \varpi}-k^{2} \phi-f=0,
\end{gathered}
$$

where we have omitted the '0' subscripts on equilibrium quantities. We have introduced the general differential operator

$$
\mathcal{D} \equiv \begin{cases}\frac{d}{d x} & \text { cartesian } \\ \frac{d}{d r}+\frac{1}{r} & \text { cylindrical }\end{cases}
$$

the coefficient functions

$$
\begin{aligned}
A_{1}= & \frac{k^{4} P^{\prime} B^{3}}{\omega^{4} \rho^{2}} \frac{d B}{d \varpi}-\frac{k^{2} B^{2}}{\omega^{2} \rho}\left[\left(\frac{B}{\rho}+\frac{3 P^{\prime}}{B}\right) \frac{d B}{d \varpi}+\left(P^{\prime \prime}-\frac{2 P^{\prime}}{\rho}\right) \frac{d \rho}{d \varpi}\right] \\
& +\left(P^{\prime \prime}-\frac{2 B^{2}}{\rho^{2}}\right) \frac{d \rho}{d \varpi}+\frac{d \psi}{d \varpi}+\frac{3 B}{\rho} \frac{d B}{d \varpi}
\end{aligned}
$$




$$
\begin{aligned}
A_{2}= & \frac{k^{4} B^{3}}{\omega^{4} \rho} \frac{d B}{d \varpi}+\frac{k^{2}}{\omega^{2}} B^{2}\left(\frac{1}{\rho} \frac{d \rho}{d \varpi}-\frac{3}{B} \frac{d B}{d \varpi}\right), \\
A_{3}= & -\frac{k^{4} B^{4}}{\omega^{6} \rho^{2}}\left(\frac{d B}{d \varpi}\right)^{2}+\frac{k^{2} B^{3}}{\omega^{4} \rho} \frac{d B}{d \varpi}\left(\frac{d^{2} B}{d \varpi^{2}} / \frac{d B}{d \varpi}+\frac{4}{B} \frac{d B}{d \varpi}-\frac{3}{\rho} \frac{d \rho}{d \varpi}-C\right) \\
& +\frac{B^{2}}{\omega^{2}}\left[k^{2}+\frac{1}{\rho} \frac{d^{2} \rho}{d \varpi^{2}}-2\left(\frac{1}{\rho} \frac{d \rho}{d \varpi}\right)^{2}-\frac{1}{B} \frac{d^{2} B}{d \varpi^{2}}-\left(\frac{1}{B} \frac{d B}{d \varpi}\right)^{2}\right. \\
& \left.+\frac{3}{\rho B} \frac{d \rho}{d \varpi} \frac{d B}{d \varpi}+C\left(\frac{1}{B} \frac{d B}{d \varpi}-\frac{1}{\rho} \frac{d \rho}{d \varpi}\right)\right]-\rho,
\end{aligned}
$$

and the cylindrical coefficient function

$$
C=\mathcal{D}-\frac{d}{d \varpi}=\left\{\begin{array}{ll}
0 & \text { cartesian } \\
\frac{1}{r} & \text { cylindrical }
\end{array} .\right.
$$

For boundary conditions, we take

$$
\begin{array}{cl}
f=1, \frac{d \phi}{d \varpi}=0, w=0 & \text { at } \varpi=0 \\
f=0, w=0 & \text { at } \varpi=\infty .
\end{array}
$$

Real molecular clouds will, of course, be pressure confined at some finite distance from the center. However, as long as this distance is larger than the scale height (in the $\varpi$ direction), the influence of the pressure confinement will be small (see Nagasawa 1987).

\section{Perturbation Solutions and Dispersion Relations}

In this section, we discuss the solutions and dispersion relations for the perturbation problem presented in the preceding section. Equations (29 31) constitute a disguised eigenvalue problem; the equations could be cast into the usual eigenvalue equation form. For numerical purposes, we choose, instead, a form which isolates terms involving the derivatives of the perturbation. Also, in this form, the possibility of a singularity becomes evident. The coefficient of $d f / d \varpi$ vanishes when

$$
\rho^{2}+\left[\kappa+B^{2}\left(1-\frac{k^{2}}{\omega^{2}}\right)\right] \rho-\kappa \frac{k^{2}}{\omega^{2}} B^{2}=0,
$$

where we have adopted the equation of state (7). In the isothermal case, a singularity occurs where

$$
\rho=B^{2}\left(\frac{k^{2}}{\omega^{2}}-1\right) .
$$


This singularity indicates that the addition of a magnetic field can preclude the existence of propagating $\left(\omega^{2}>0\right)$ wave solutions that are localized in the filament. This absence of propagating waves occurs because Alfvén waves on the uniform magnetic field background can easily carry the energy of a propagating wave away from the filament, causing the wave to decay in time. We avoid this complication by solving the problem only in the unstable regime, where $\omega^{2}<0$, and hence no singularity arises.

For the marginally stable perturbation, equations (29 31) can be simplified by applying the conditions $\omega^{2}=0$ and $w \equiv 0$. When the equilibrium magnetic field strength $B$ is uniform, we find

$$
\begin{aligned}
P^{\prime} f_{0}+\rho \phi_{0} & =0, \\
\mathcal{D} \frac{d \phi_{0}}{d \varpi}+\frac{\rho}{P^{\prime}} \phi_{0} & =k_{0}^{2} \phi_{0} .
\end{aligned}
$$

We solve this well known equation to determine the critical wavenumber $k_{0}$ and associated marginally stable perturbation functions, $f_{0}$ and $\phi_{0}$.

Once we have the marginally stable solution, we proceed to smaller values for the wavenumber $k$. We use a relaxation algorithm with a finite difference approximation (Press et al. 1992) to solve equations (29 31) for successively smaller values of $k^{2}$, where the solution at the previous step is used as an initial approximation.

\subsection{Filaments}

First, we consider the case of molecular cloud filaments. We adopt cylindrical coordinates $(r, \phi, z)$ and assume azimuthal symmetry. We use the one-parameter turbulent equation of state (7). Notice that the limit of vanishing turbulence parameter $\kappa \rightarrow 0$ corresponds to a purely isothermal cloud. The opposite limit, $\kappa \rightarrow \infty$, corresponds to a highly turbulent cloud with a purely logatropic equation of state $p=\log \rho$ (see Appendix A for details of the differences in the scaling of variables for the purely logatropic case). Observational considerations indicate that the range $6<\kappa<50$ is the most physically relevant. We also assume that the unperturbed magnetic field is uniform. Using typical values for the observed characteristics of molecular clouds, $B \sim 10 \mu \mathrm{G}, \rho \sim 4 \cdot 10^{-20} \mathrm{~g} \mathrm{~cm}^{-3}$, and $c_{\mathrm{s}} \sim 0.20 \mathrm{~km} \mathrm{sec}^{-1}$, we find that the physically interesting magnitude of the dimensionless field strength variable is $B \sim 0.7$.

All the dispersion relations possess the same general form and exhibit a minimum value of $\omega^{2}$ at a certain wavenumber, which we will call $k_{\text {fast }}$. Since $\omega^{2}<0$ throughout the region that we explore, all solutions that we find represent unstable modes. The magnitude of the frequency $|\omega|$ is the rate of growth for a particular mode, and hence the minimum of the dispersion relation corresponds to the fastest growing mode. The location of this point 
$\left(k_{\text {fast }}, \omega_{\text {fast }}^{2}\right)$ in the dispersion plane varies as the parameters $\kappa$ and $B$ change. In Figure 1a, we show isothermal $(\kappa=0)$ dispersion relations for various values of the equilibrium field strength $B$. These results agree well with corresponding results of Nagasawa (1987), which were obtained using a different numerical technique. Notice that the magnetic field tends to decrease the instability of all unstable modes but has no influence on the critical wavenumber and has only a very small effect on the fast wavenumber $k_{\text {fast }}$. This effect is contrary to that exhibited by models which adopt a non-uniform unperturbed magnetic field. Nakamura et al. (1993) show that an isothermal filament with a constant ratio of magnetic pressure to thermal pressure is destabilized by increasing the strength of the magnetic field.

Figure 2a shows dispersion relations for various values of the turbulence parameter $\kappa$ with no magnetic field. Notice that the wavenumber has been scaled by the factor $(1+\kappa)^{1 / 2}$, which is just the effective sound speed $\partial P / \partial \rho$ at the center of the cloud. This scaling is done to account for the fact that the variable transformation performed to eliminate units uses the thermal sound speed. Displayed this way, the dispersion relations can be seen to converge to the limiting case where $p=\log \rho$ as the turbulence parameter $\kappa$ increases. Again, these results agree well with corresponding results in Paper 1. Contrary to the effect of the magnetic field, the presence of turbulence significantly increases the growth rate of the fast mode as well as decreases its scaled wavenumber. In addition, turbulence is seen to decrease the scaled critical wavenumber. Paper 1 closely examines these effects for turbulent clouds without magnetic fields.

Figures [1 b and 2/b similarly show more dispersion relations where one parameter is varied while the other is held fixed. In these figures, however, the fixed parameter (the field strength $B$ or the turbulence parameter $\kappa$ ) has a more physically relevant value. Notice in Figure [ $\mathrm{b}$ that the magnetic field is not as effective at decreasing the instability as in the isothermal case (Figure 1a). This result can be understood by again considering the effective sound speed. The dimensionless magnetic field strength variable scales inversely with the thermal sound speed. A more analytically convenient variable would use the effective sound speed instead of the thermal sound speed. We choose to use the thermal sound speed in our transformation because of its physical significance; the thermal sound speed is well determined in observations of molecular clouds. The decreased effectiveness of the magnetic field is shown clearly in Figure 3, which plots the growth rate of the fastest growing mode versus the equilibrium field strength for a variety of values of the turbulence parameter. For the isothermal filament, the effect of the magnetic field saturates around a field strength of $B \sim 2$, whereas a field strength of $B \gtrsim 10$ is required to achieve the maximum effect for the turbulent filament with $\kappa=10$.

The wavenumber of the fastest growing mode defines a length scale $\lambda_{\text {fast }}=2 \pi / k_{\text {fast }}$ 
for the fragmentation of the filament. This physical length scale is tabulated in Table 1 for several physically interesting values of the magnetic field strength and turbulence parameter. Notice that the influence of turbulence dominates the effect of the magnetic field. For isothermal filaments, a corresponding mass scale of fragmentation is set by

$$
M_{\text {frag }}=8 \pi \lambda_{\text {fast }} .
$$

Paper 1 showed that the physical mass scale for the isothermal filament is given by

$$
M_{\text {frag }} \approx 14.5 M_{\odot}\left[\frac{c_{s}}{0.20 \mathrm{~km} / \mathrm{s}}\right]^{3}\left[\frac{\rho_{c}}{4 \times 10^{-20} \mathrm{~g} / \mathrm{cm}^{3}}\right]^{-1 / 2} .
$$

Here, in the strong magnetic field limit, we find this mass scale to be slightly decreased;

$$
M_{\text {frag }} \approx 13.5 M_{\odot}\left[\frac{c_{s}}{0.20 \mathrm{~km} / \mathrm{s}}\right]^{3}\left[\frac{\rho_{c}}{4 \times 10^{-20} \mathrm{~g} / \mathrm{cm}^{3}}\right]^{-1 / 2} .
$$

Since the mass per unit length of turbulent filaments is formally infinite, so is their fragmentation mass scale.

Measuring the actual physical spacing of clumps along a molecular cloud is rather difficult, since the uncertainty in the distance and orientation of the cloud is often large. A more easily measured quantity is the aspect ratio

$$
\mathcal{F}=\frac{\lambda_{\text {fast }}}{R_{\mathrm{HWHM}}},
$$

where $R_{\text {HWHM }}$ is the half-width at half-maximum of the filament. The dependency of this ratio on the parameters $\kappa$ and $B$ is illustrated in Figure 6 . Notice that the effect of the magnetic field on the aspect ratio $\mathcal{F}$ is rather small for isothermal clouds compared to the effect on turbulent clouds. We also note that there is a threshold field strength, below which there is little change in $\mathcal{F}$ and above which the influence of the magnetic field increases dramatically.

Figures 5 and 6 show cross-sections of the perturbed density and the flow of the fluid and the magnetic field lines for various filaments. Isothermal filaments with and without magnetic field are shown in Figure 5; similarly, turbulent filaments are shown in Figure 6. These figures illustrate the physical reason for the limited effectiveness of the magnetic field. For $B=1$, the effect of the magnetic field in the isothermal filament is near saturation. This saturation is manifested by the nearly axial flow of the fluid. In contrast, when the magnetic field is absent the flow has a significant radial component. Notice that this contrast is not present in the turbulent filament, where the effect of the magnetic field at $B=1$ is still far from saturation. These results support the conclusion that the saturation occurs because the magnetic field cannot prevent contraction of the fluid along the field lines (Nagasawa 1987). 


\subsection{Sheets}

Now, we consider a molecular cloud sheet or stratified layer. We adopt cartesian coordinates $(x, y, z)$ and assume translational symmetry in the $y$-direction. Here, $\varpi=x$, the differential operator $\mathcal{D}$ is just a derivative, and the cylindrical coefficient function $C$ is identically zero. The problem is solved using the same numerical technique as used for the filament.

The dispersion relations for the sheet (Figures 7 and 8) possess the same general form and exhibit similar dependencies on the magnetic field and turbulence parameters as those for the filament. In general, the growth rates are about 30-40\% larger for the sheet than for the filament. The influence of the magnetic field and turbulence on the fastest growth rate is displayed in Figure 9. Notice that these effects are qualitatively the same as for the filament discussed in the previous section. The magnetic field tends to stabilize the cloud. As before with filaments, this effect is contrary to that exhibited in isothermal sheets with a constant ratio of magnetic pressure to thermal pressure (Nakano 1988; Nakamura, Hanawa, \& Nakano 1991).

We tabulate the physical fragmentation length scale $\lambda_{\text {fast }}$ in Table 2. As before, for the isothermal filament, this length scale yields a mass scale of fragmentation for the isothermal sheet. The magnetic field, however, does not significantly affect the the length scale of the isothermal sheet and, hence, has little effect upon the corresponding mass scale (see Paper 1 for a discussion of the mass scales).

\section{Discussion \& Summary}

In this paper, we have studied gravitational instabilities of astrophysical fluids in two spatial dimensions. Although many of the results are general, molecular clouds provide our primary motivation. We have accounted for the presence of turbulence in molecular clouds by adding a logarithmic term in the equation of state and included the influence of a uniform axial magnetic field perfectly frozen in the fluid. We have studied wave-like perturbations on the hydrostatic equilibrium states and have numerically determined the dispersion relations and the structure of the perturbations in the unstable regime. 


\subsection{Summary of Results}

We have found a number of results concerning gravitational instabilities in molecular clouds, which add to our general understanding of fluid dynamics in self-gravitating systems with uniform magnetic fields. Our main results can be summarized as follows:

1. The dispersion relations for these two dimensional perturbations have the same general form for all values of magnetic field strength and for all equations of state considered here. In particular, the growth rate for gravitational instability obtains a maximum value for one particular wavelength of the perturbation (see Figures 11 and 2).

2. Turbulence increases $(\sim 22 \%)$ the growth rate of the fastest growing mode when the magnetic field is not strong (see Figures 2 and 3 ).

3. Turbulence increases the length scale of the fastest growing mode when the magnetic field is not strong. Furthermore, turbulence increases the aspect ratio $\mathcal{F}$ of the structure by about $29 \%$ (see Figures 2 and 4 ).

4. A magnetic field tends to decrease the growth rate of all unstable perturbations (see Figures 1 and 3). The growth rate of the fastest growing mode is suppressed by about $12 \%$ in the absence of turbulence. This suppression is much stronger $(\sim 31 \%)$ in turbulent clouds. Since the fastest growth rate is significantly larger, however, for turbulent clouds without magnetic fields, the growth rate is nearly the same for isothermal and turbulent clouds in the strong field limit.

5. A magnetic field has a relatively small influence $(\sim 6 \%)$ on the length scale of fragmentation for isothermal clouds. For turbulent clouds, there is little effect on the length scale unless the magnetic field exceeds a threshold, which is of the order of the turbulence parameter. Above this threshold, the magnetic field reduces the fragmentation length scale of turbulent clouds up to about $15 \%$ (see Figure 4).

6. In certain regimes, turbulence and magnetic field effects counteract one another, in the sense that turbulence enhances the growth while the magnetic field suppresses the growth. For example, a molecular cloud filament with $\kappa=10$ and $B=2$ has essentially the same fragmentation rate $\left|\omega_{\text {fast }}\right|$ as a filament with $\kappa=0=B$ (see Figure [3).

7. The mass scale for fragmentation of isothermal filaments depends only weakly on the magnetic field strength and typically has a value of $\sim 14 M_{\odot}$, which is much larger than a typical stellar mass. The mass scale for a turbulent filament is formally infinite (see also Paper 1). 
8. The dispersion relations for molecular cloud sheets are qualitatively similar to those of filamentary clouds. Moreover, the fragmentation rate $\left|\omega_{\text {fast }}\right|$ and length scale $\lambda_{\text {fast }}$ depend on the turbulence parameter $\kappa$ and the magnetic field strength $B$ in the same way as in the case of the filament (compare Figures 9 and 3 ).

\subsection{Implications for Observed Molecular Clouds}

The results of this paper can be compared with observed structures in molecular clouds. In particular, these theoretical calculations make several definite predictions that can be used to further our understanding of the physics of molecular cloud systems. These predictions are outlined below.

For given values of the turbulence parameter $\kappa$ and the magnetic field strength $B$, the dispersion relations predict that gravitational fragmentation occurs with a well defined length scale $\lambda_{\text {fast }}$ and has a well defined growth rate $\left|\omega_{\text {fast }}\right|$. The length scale $\lambda_{\text {fast }}$ can be deduced from observed molecular clouds maps and compared with these theoretical results. If the turbulence parameter $\kappa$ can be independently determined from observations of molecular line-widths, and if the magnetic field strength $B$ can also be measured, then one can obtain a direct test of the theory.

Unfortunately, however, observational uncertainties often prevent a clean determination of the length scale $\lambda_{\text {fast }}$. These uncertainties arise from the (often unknown) projection angle and the (imprecisely known) distance to the cloud. This latter effect can be avoided by considering the aspect ratio $\mathcal{F}=\lambda_{\text {fast }} / R_{\text {HWHM }}$ of the perturbation. This ratio is shown as a function of magnetic field strength $B$ and the turbulence parameter $\kappa$ in Figure 4 .

Observations indicate that the strength of the magnetic field in actual molecular clouds increases with increasing mass density (Troland \& Heiles 1986; Heiles et al. 1993), rather than remaining constant throughout the cloud as we have assumed in our model. Since the equilibrium configuration of such clouds are supported in part by the magnetic field, this difference can lead to qualitative changes in the dependence of the fast mode on the strength of the field. For example, isothermal models that adopt a constant ratio of magnetic to thermal pressures, i.e., $B \propto \rho^{1 / 2}$, show that the magnetic field destabilizes the cloud and decreases the length scale of fragmentation (Nakamura, Hanawa, \& Nakano 1993; Nakamura, Hanawa, \& Nakano 1991). Several reasonably successful efforts to apply such models to particular clouds have been made (Hanawa et al. 1993; Nakamura, Hanawa, \& Nakano 1993; Matsumoto, Nakamura, \& Hanawa 1994).

Another important observational diagnostic is the structure of the velocity field. Paper 
showed that unstable (i.e., growing) perturbations have a completely different velocity field signature than propagating waves. Furthermore, as we show in this paper, the velocity field of the perturbations depends on both the magnetic field strength and the turbulence parameter (see Figures 5 and 6). In particular, as the magnetic field strength increases, the radial velocities become smaller and the flow is almost entirely in the $z$ direction (along the filament - see Figure 5). Observations of the geometry of the velocity field in molecular cloud filaments can thus be a powerful diagnostic of the internal dynamics.

\subsection{Directions for Future Work}

In this paper, we have begun to study the evolution of structure in molecular clouds. Numerous directions for future work, however, remain to be explored. These directions include both theoretical and observational studies.

Small scale magnetohydrodynamic motions, such as Alfvén waves, are often considered to account for the turbulence observed in molecular clouds. Here, for the first time, we have attempted to study the effects of this turbulence in conjunction with the effects of large scale magnetic fields, which are widely believed to provide a significant fraction of pressure support for molecular clouds. Our model, however, assumed a uniform unperturbed magnetic field, which provides no support to the equilibrium state of the cloud but does impede the fragmentation of the cloud by gravitational instability. A more relevant unperturbed configuration should include a magnetic field which does contribute to the support of the equilibrium state. Observations of molecular clouds suggest that the field strength often varies with the density roughly according to $B \sim \rho^{1 / 2}$ (e.g., see Troland \& Heiles 1986 and Heiles et al. 1993). Furthermore, this relation is theoretically attractive since it implies a constant ratio of magnetic to thermal energy. Stodółkiewicz (1963) determined the critical length scale $\left(2 \pi / k_{0}\right)$ for such an isothermal system. Though several studies on isothermal clouds with such an unperturbed magnetic field have been performed Nakamura, Hanawa, \& Nakano 1993; Nakamura, Hanawa, \& Nakano 1991), the effects of turbulence in the presence of such a field have yet to be investigated.

In future work, it will be interesting to investigate the behavior of perturbations using a wider variety of equations of state. We have focused here on equations of state that are softer than isothermal; stiffer equations of state should also be examined. For these equations of state, the equilibrium configurations of filaments have a finite radius, outside of which the density is zero. Other functional forms for the equation of state of a turbulent gas should be considered as well. The logarithmic term used in this work was deduced empirically. Theoretical studies of turbulence in molecular clouds may lead to different formulations for 
the turbulent contribution to the pressure.

Finally, we note that the clumps observed in molecular cloud filaments are generally not small perturbations on an equilibrium filament. In general, molecular cloud substructure lies in the fully nonlinear regime. In previous work, we began to study nonlinear waves in molecular clouds (e.g., Adams, Fatuzzo, \& Watkins 1994; see also Infeld \& Rowlands 1990). This work was restricted to one spatial dimension, although two-dimensional effects were heuristically taken into account. Future work should study two-dimensional wave motions and instabilities in the fully nonlinear regime.

We would like to thank Greg Laughlin, Marco Fatuzzo, Lee Hartmann, Phil Myers, Paul Ho, and Jennifer Wiseman for constructive comments and discussion. This work was supported by a NSF Young Investigator Award, NASA Grant No. NAG 5-2869, and by funds from the Physics Department at the University of Michigan.

\section{A. Transformation to Dimensionless Variables}

The equations of motion of the physical fields of a fluid with a perfectly frozen magnetic field can be written as

$$
\begin{gathered}
\frac{\partial \rho}{\partial t}+\nabla \cdot(\rho \mathbf{u})=0 \\
\rho \frac{\partial \mathbf{u}}{\partial t}+\rho(\mathbf{u} \cdot \nabla) \mathbf{u}+\nabla p+\rho \nabla \psi-\frac{1}{4 \pi}(\nabla \times \mathbf{B}) \times \mathbf{B}=0 \\
\frac{\partial \mathbf{B}}{\partial t}+\nabla \times(\mathbf{B} \times \mathbf{u})=0 \\
\nabla^{2} \psi=4 \pi G \rho
\end{gathered}
$$

where $\rho, \mathbf{u}, p$, and $\psi$ are the mass density, velocity, pressure, and gravitational potential of the fluid, respectively, and $\mathbf{B}$ is the magnetic field. To simplify the problem, we transform to dimensionless variables according to

$$
\begin{array}{ccc}
t \rightarrow \hat{t} t & \rho \rightarrow \hat{\rho} \rho & \mathbf{u} \rightarrow \hat{u} \mathbf{u} \\
\mathbf{x} \rightarrow \hat{t} \hat{u} \mathbf{x} & p \rightarrow \hat{\rho} \hat{u}^{2} p & \mathbf{B} \rightarrow(4 \pi \hat{\rho})^{1 / 2} \hat{u} \mathbf{B} \\
& \psi \rightarrow \hat{u}^{2} \psi &
\end{array}
$$

where $\hat{t} \equiv(4 \pi G \hat{\rho})^{-1 / 2}$. Throughout this paper, we let $\hat{\rho}=\rho_{\mathrm{c}}$, the central density of the equilibrium state. For cases where the equation of state takes the form of equation (7), we let $\hat{u}=c_{\mathrm{s}}$, the thermal sound speed. When we consider the purely logatropic equation of state $p=\hat{p} \log (\rho / \hat{\rho})$, we set $\hat{u}=(\hat{p} / \hat{\rho})^{1 / 2}$. Under the transformation (A5), the fluid equations (A1 A4) are cast into the form (11 4 ). 
Table 1. Physical Fragmentation Length Scale for Filament

\begin{tabular}{cccccc}
\hline \hline $\begin{array}{c}\text { Magnetic Field } \\
B\end{array}$ & 0 & 1 & 5 & 10 & 20 \\
\hline 0 & 0.777 & 1.51 & 2.89 & 3.99 & 5.57 \\
0.5 & 0.752 & 1.52 & 2.93 & 4.02 & 5.59 \\
1.0 & 0.738 & 1.42 & 2.96 & 4.09 & 5.65 \\
2.0 & 0.731 & 1.31 & 2.74 & 4.01 & 5.74 \\
10 & 0.729 & 1.26 & 2.39 & 3.32 & 4.69 \\
\hline
\end{tabular}

Note. - The values in this table give the fragmentation length scale in parsecs. The values scale with the thermal sound speed and mass density according to $\lambda \sim$ $\left(c_{\mathrm{s}} / 0.20 \mathrm{~km} \mathrm{sec}^{-1}\right)\left(\rho_{\mathrm{c}} / 4.0 \times 10-20 \mathrm{~g} \mathrm{~cm}^{-3}\right)^{-1 / 2}$.

Table 2. Physical Fragmentation Length Scale for Sheet

\begin{tabular}{cccccc}
\hline \hline \multirow{2}{*}{$\begin{array}{c}\text { Magnetic Field } \\
B\end{array}$} & 0 & \multicolumn{5}{c}{ Turbulence parameter $\kappa$} \\
\hline 0 & 0.677 & 1.10 & 2.02 & 2.76 & 3.84 \\
0.5 & 0.677 & 1.12 & 2.03 & 2.78 & 3.85 \\
1.0 & 0.677 & 1.13 & 2.07 & 2.81 & 3.87 \\
2.0 & 0.675 & 1.12 & 2.10 & 2.87 & 3.95 \\
10 & 0.673 & 1.08 & 1.99 & 2.75 & 3.87 \\
\hline
\end{tabular}

Note. - The values in this table give the fragmentation length scale in parsecs. The values scale with the thermal sound speed and mass density according to $\lambda \sim$ $\left(c_{\mathrm{s}} / 0.20 \mathrm{~km} \mathrm{sec}^{-1}\right)\left(\rho_{\mathrm{c}} / 4.0 \times 10-20 \mathrm{~g} \mathrm{~cm}^{-3}\right)^{-1 / 2}$. 


\section{REFERENCES}

Adams, F. C., Lada, C. J., \& Shu, F. H. 1987, ApJ, 312, 788

Adams, F. C., Fatuzzo, M., \& Watkins, R. 1994, ApJ, 426, 629

Arons, J., \& Max, C. 1975, ApJ, 196, L77

Blitz, L. 1993, in Protostars and Planets III, ed. E. Levy \& M. S. Mathews (Tucson: Univ. of Arizona Press), 125

Butner, H. M., Evans, N. J., Lester, D. F., Levreault, R. M., \& Strom, W. E. 1991, ApJ, 376,676

Dame, T. M., Elmegreen, B. G., Cohen, R. S., \& Thaddeus, P. 1986, ApJ, 305, 892

de Geus, E. J., Bronfman, L., \& Thaddeus, P. 1990, A\&A, 231, 137

Dewer, R. L. 1970, Phys. Fluids, 13, 2710

Dutrey, A., Langer, W. D., Bally, J., Duvert, G., Castets, A., \& Wilson, R. W. 1991, A\&A, 247, L9

Elmegreen, B. G. 1990, ApJ, 361, L77

Fatuzzo, M., \& Adams, F. C. 1993, ApJ, 412, 146

Gehman, C. S., Adams, F. C., Fatuzzo, M., \& Watkins, R. 1996, ApJ, 457, 718 (Paper I)

Hanawa, T., et al. 1993, ApJ, 404, L83

Hartmann, L., Boss, A., Calvet, N., \& Whitney, B. 1994, ApJ, 430, L49

Heiles, C., Goodman, A. A., McKee, C. F., \& Zweibel, E. G. 1993, in Protostars and Planets III, ed. E. Levy \& J. Lunine (Tucson: University of Arizona Press), p. 279

Houlahan, P., \& Scalo, J. M. 1992, ApJ, 393, 172

Infeld, E., \& Rowlands, G. 1990, Nonlinear Waves, Solitons, and Chaos (Cambridge: Cambridge Univ. Press)

Inutsuka, S., \& Miyama, S. M. 1993, ApJ, 388, 392

Jeans, J. H. 1928, Astronomy and Cosmogony (Cambridge: Cambridge Univ. Press)

Kenyon, S. J., Calvet, N., \& Hartmann, L. 1993, ApJ, 414, 676 
Langer, W. D. 1978, ApJ, 225, 95

Larson, R. B. 1972, MNRAS, 157, 121

Larson, R. B. 1981, MNRAS, 194, 809

Larson, R. B. 1985, MNRAS, 214, 379

Lizano, S., \& Shu, F. H. 1989, ApJ, 342, 834

Matsumoto, T., Nakamura, F., \& Hanawa, T. 1994, PASJ, 46, 243

McBreen, B., Fazio, G. G., Stier, M., \& Wright, E. L. 1979, ApJ, 232, L183

McKee, C. F., \& Zweibel, E. G. 1995, ApJ, 440, 686

Miyama, S. M., Narita, S., \& Hayashi, C. 1987 Prog. Theor. Phys., 78, 1051

Myers, P. C. 1983, ApJ, 270, 105

Myers, P. C. 1991, in IAU Symp. 147, Fragmentation of Molecular Clouds and Star Formation, ed. E. Falgarone \& G. Duvert (Dordrecht: Kluwer), 221

Myers, P. C., \& Fuller, G. A. 1992, ApJ, 396, 631

Myers, P. C., \& Goodman, A. A. 1988, ApJ, 329, 392

Nagasawa, M. 1987, Prog. Theor. Phys., 77, 635

Nakamura, F., Hanawa T., \& Nakano, T. 1991, PASJ, 43, 685

Nakamura, F., Hanawa T., \& Nakano, T. 1993, PASJ, 45, 551

Nakamura, F., Hanawa T., \& Nakano, T. 1995, ApJ, 444, 770

Nakano, T. 1988, PASJ, 40, 593

Norman, C., \& Silk, J. 1980, ApJ, 238, 158

Ostriker, J. 1964, ApJ, 140, 1056

Press, W. H., Teukolsky, S. A., Vetterling, W. T., \& Flannery, B. P. 1992, Numerical Recipes in C: The Art of Scientific Computing (2nd ed.; Cambridge: Cambridge Univ. Press)

Pudritz, R. E. 1990, ApJ, 350, 195

Schneider, S., \& Elmegreen, B. 1979, ApJS, 41, 87 
Shu, F. H. 1977, ApJ, 214, 488

Shu, F. H. 1992, Gas Dynamics (Mill Valley: University Science)

Shu, F. H., Adams, F. C., \& Lizano, S. 1987, ARA\&A, 25, 23

Solomon, P. M., Rivolo, A. R., Barrett, J. W., \& Yahil, A. 1987, ApJ, 319, 730

Spitzer, L. 1942, ApJ, 95, 329

Stodółkiewicz, J. S. 1963, Acta Astronomica, 13, 31

Terebey, S., Shu, F. H., \& Cassen, P. 1984, ApJ, 286, 529

Troland, T. H., \& Heiles, C. 1986, ApJ, 301, 339

Wiseman, J. J., \& Adams, F. C. 1994, ApJ, 435, 708

Wiseman, J. J. 1996, Harvard Univ. Ph.D. thesis 


\section{Figure Captions}

Fig. 1.- Dispersion relations for molecular cloud filaments. The abscissa is the wave number multiplied by the effective sound speed, and the ordinate is the square of the frequency. For any particular wave number in the region plotted, the growth rate $(|\omega|)$ decreases with increasing magnetic field strength. (a) Isothermal filament $(\kappa=0)$ with magnetic field strengths of $0,0.05,0.10,0.20,0.50,1.0$, and 2.0. (b) Turbulent filament $(\kappa=10)$ with magnetic field strengths of $0,0.20,0.50,1.0,2.0,5.0$, and 10 .

Fig. 2.- Dispersion relations for molecular cloud filaments. Solid curves show isothermal dispersion relations. The abscissa is the wave number multiplied by the effective sound speed at the center of the filament. The ordinate is the square of the frequency. Dispersion relations are shown for various values of the turbulence parameter $\kappa$. Notice that the location of the minimum moves to larger growth rates and smaller wavenumbers as the turbulence parameter $\kappa$ increases. (a) Filament with no magnetic field. $\kappa=0,1,2,5,10, \infty$. (b) Magnetic filament $(B=1) . \kappa=0,1,2,5,10,20$.

Fig. 3.- Growth rate of the fastest growing mode. Points represent minima of dispersion relations for various magnetic field and turbulence strengths. Points for fixed values of the turbulence parameter $(\kappa=0,1,2,5,10,20)$ are interpolated with cubic splines.

Fig. 4.- Relative length scale of fragmentation. Points represent minima of dispersion relations for various magnetic field and turbulence strengths. The ordinate is the aspect ratio, the wavelength of the fastest growing mode divided by the half-width at half-maximum of the unperturbed filament. Points for fixed values of the turbulence parameter $(\kappa=0,1$, $2,5,10,20)$ are interpolated with cubic splines.

Fig. 5.- Cross-section of the fastest growing mode in isothermal filaments without and with magnetic field ( $B=0,1)$. The total density (equilibrium plus perturbation) is represented with solid contours $(\rho=0.2,0.4,0.6,0.8,1.0,1.2)$, and the fluid velocity is represented by the array of arrows on the right half of the filament. When present, magnetic field lines are shown as dashed lines on the left side.

Fig. 6. - Cross-section of the fastest growing mode in turbulent filaments $(\kappa=10)$ without and with magnetic field $(B=0,1)$. The total density (equilibrium plus perturbation) is represented with contours $(\rho=0.2,0.4,0.6,0.8,1.0,1.2)$, and the fluid velocity is represented by the array of arrows on the right half of the filament. When present, magnetic field lines are shown as dashed lines on the left side. 
Fig. 7.- Dispersion relations for turbulent molecular cloud sheets with magnetic field strengths of $0,0.5,1.0,2.0,5.0$, and 10. The turbulence parameter $\kappa=10$ is fixed. For any particular wave number, the growth rate $(|\omega|)$ decreases with increasing magnetic field strength.

Fig. 8.- Dispersion relations for molecular cloud sheets with a fixed magnetic field strength of $B=1.0$ and various degrees of turbulence $(\kappa=0,1,2,5,10,20)$. The abscissa is the wave number multiplied by the effective sound speed at the center of the filament. The fastest growth rate $\left(\left|\omega_{\text {fast }}\right|\right)$ increases with increasing values of the turbulence parameter $\kappa$.

Fig. 9.- Growth rate of the fastest growing mode in the sheet. Points represent minima of dispersion relations for various magnetic field and turbulence strengths. Points for fixed values of the turbulence parameter $(\kappa=0,1,2,5,10,20)$ are interpolated with cubic splines. 


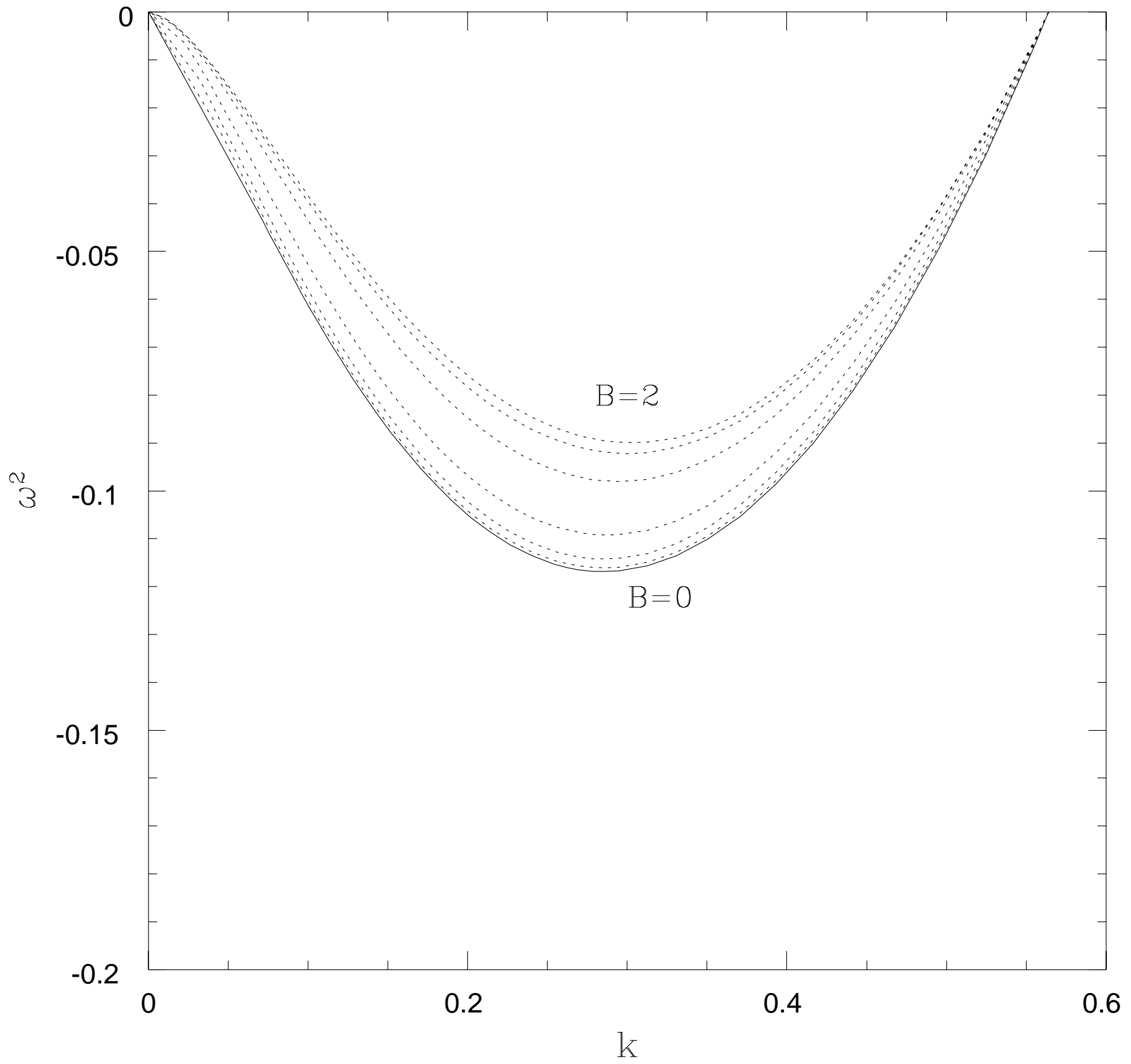

Figure 1a 


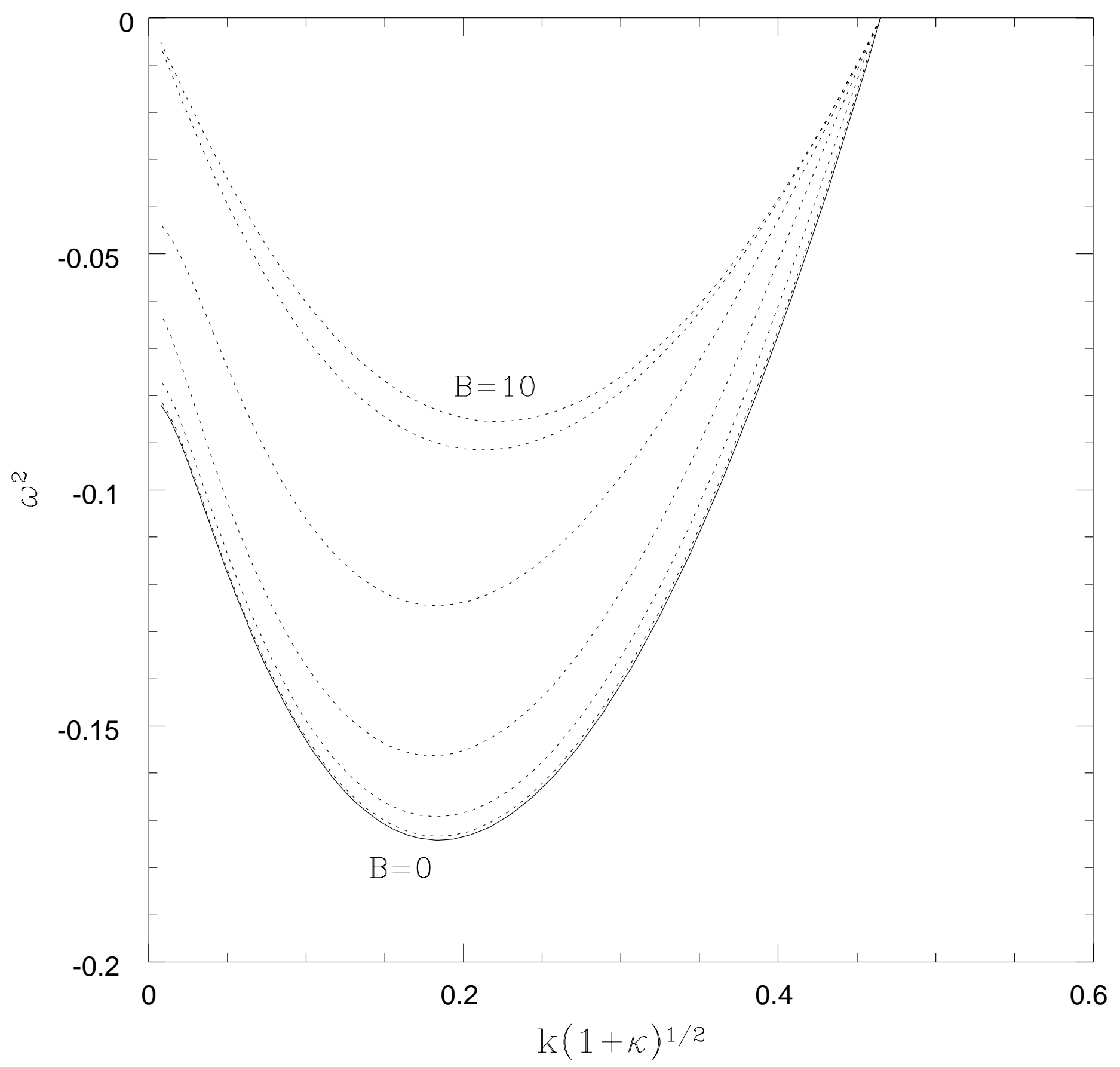

Figure 1b 


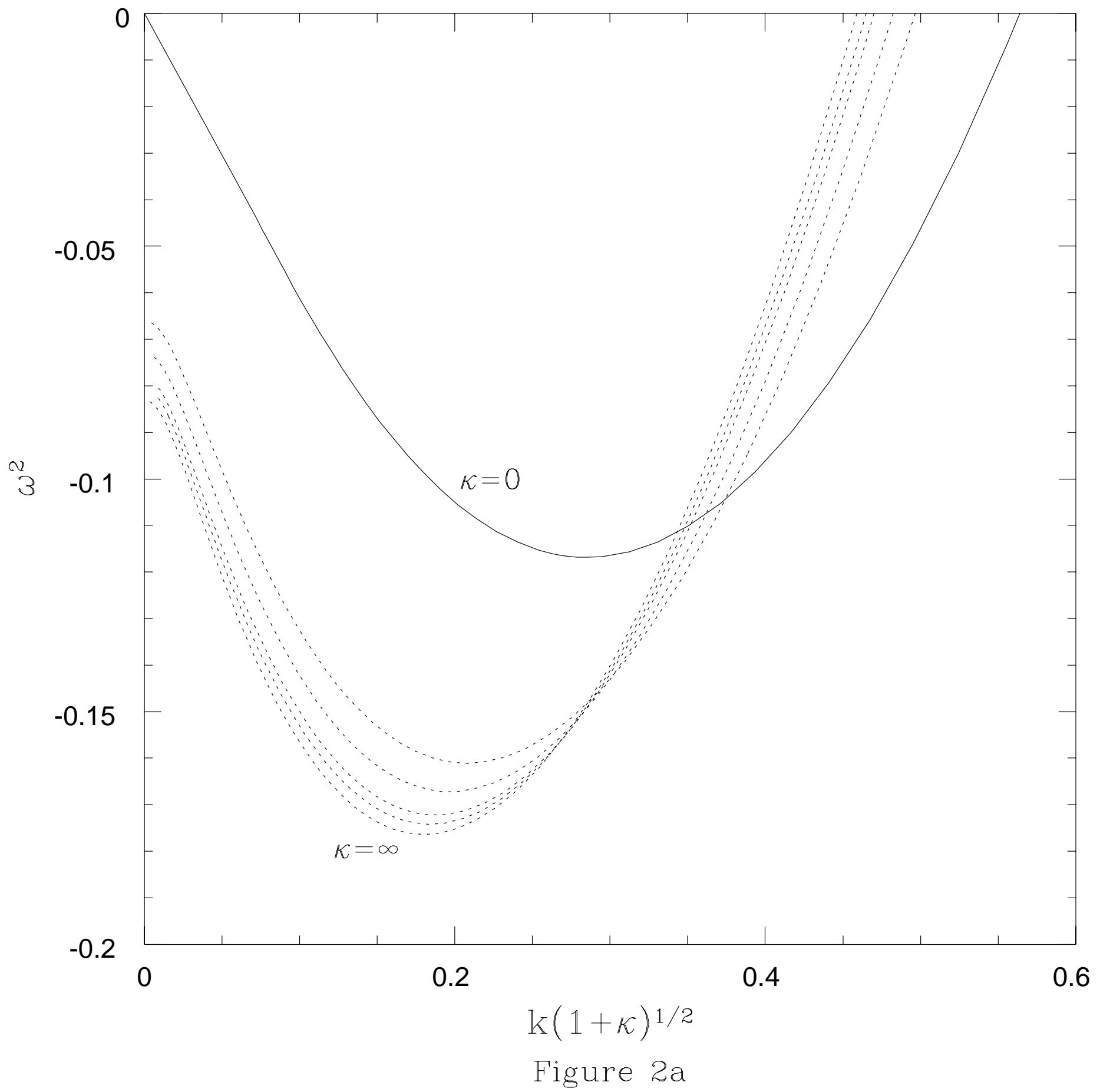




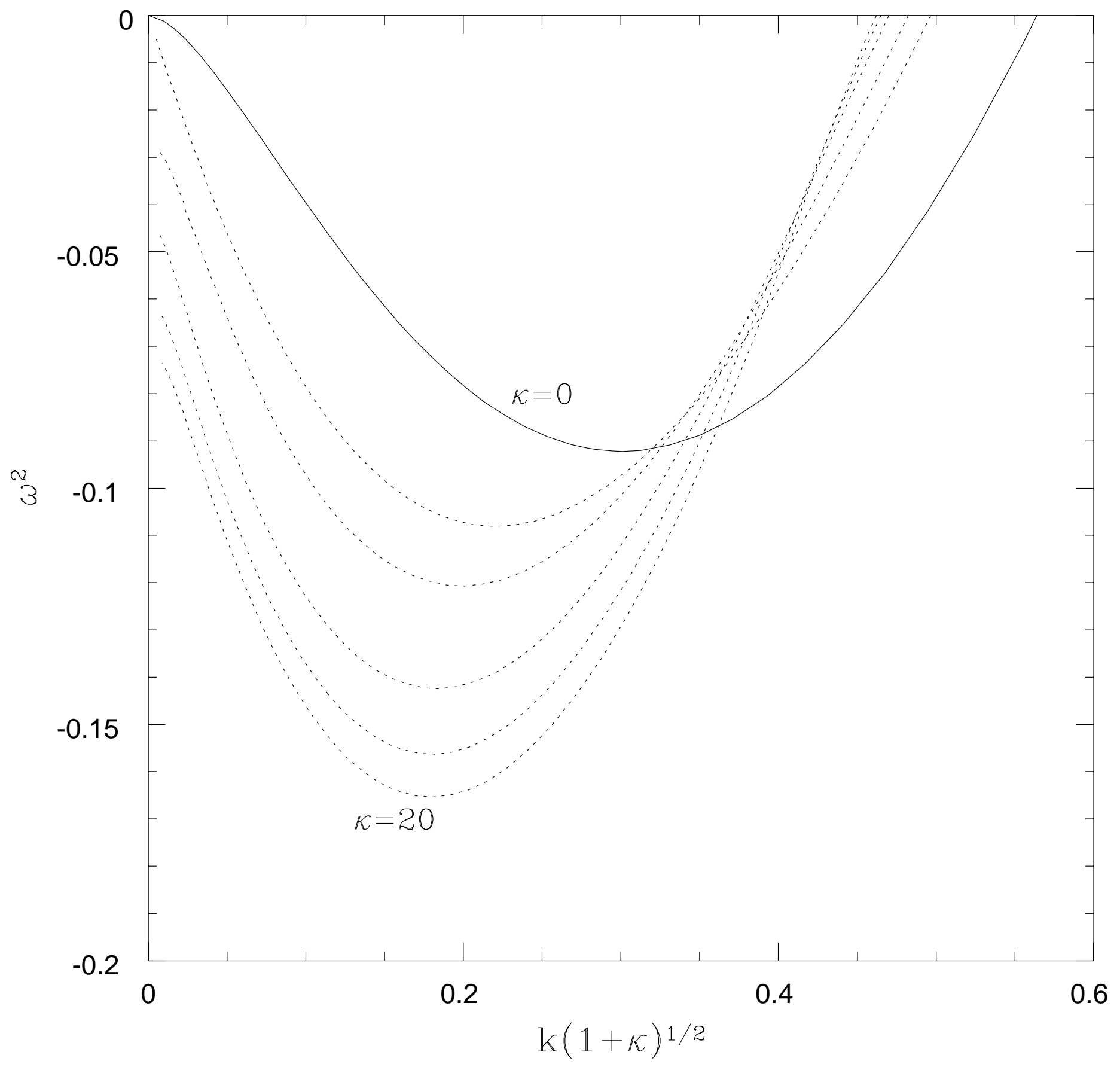

Figure 2b 


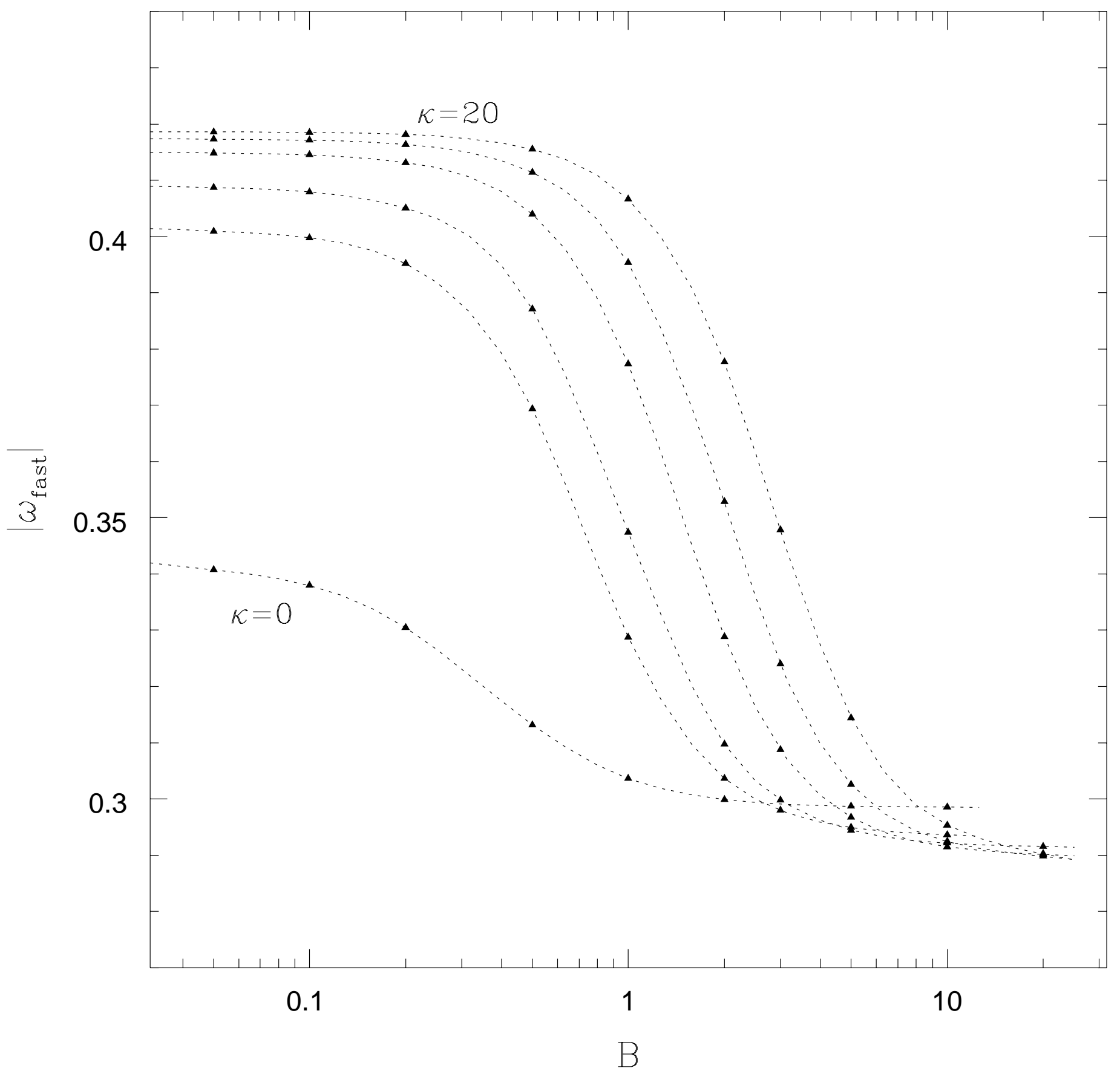

Figure 3 


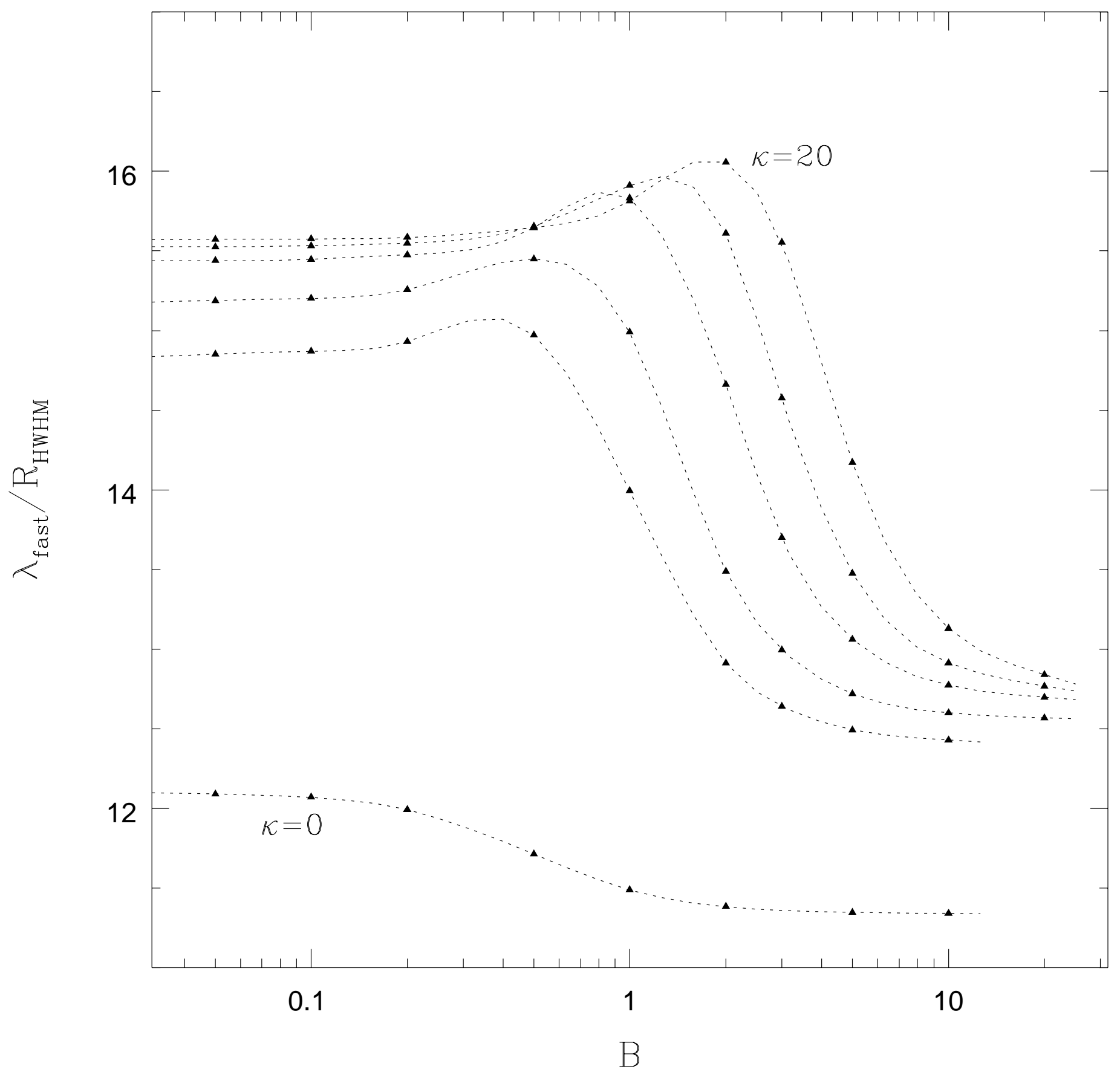

Figure 4 


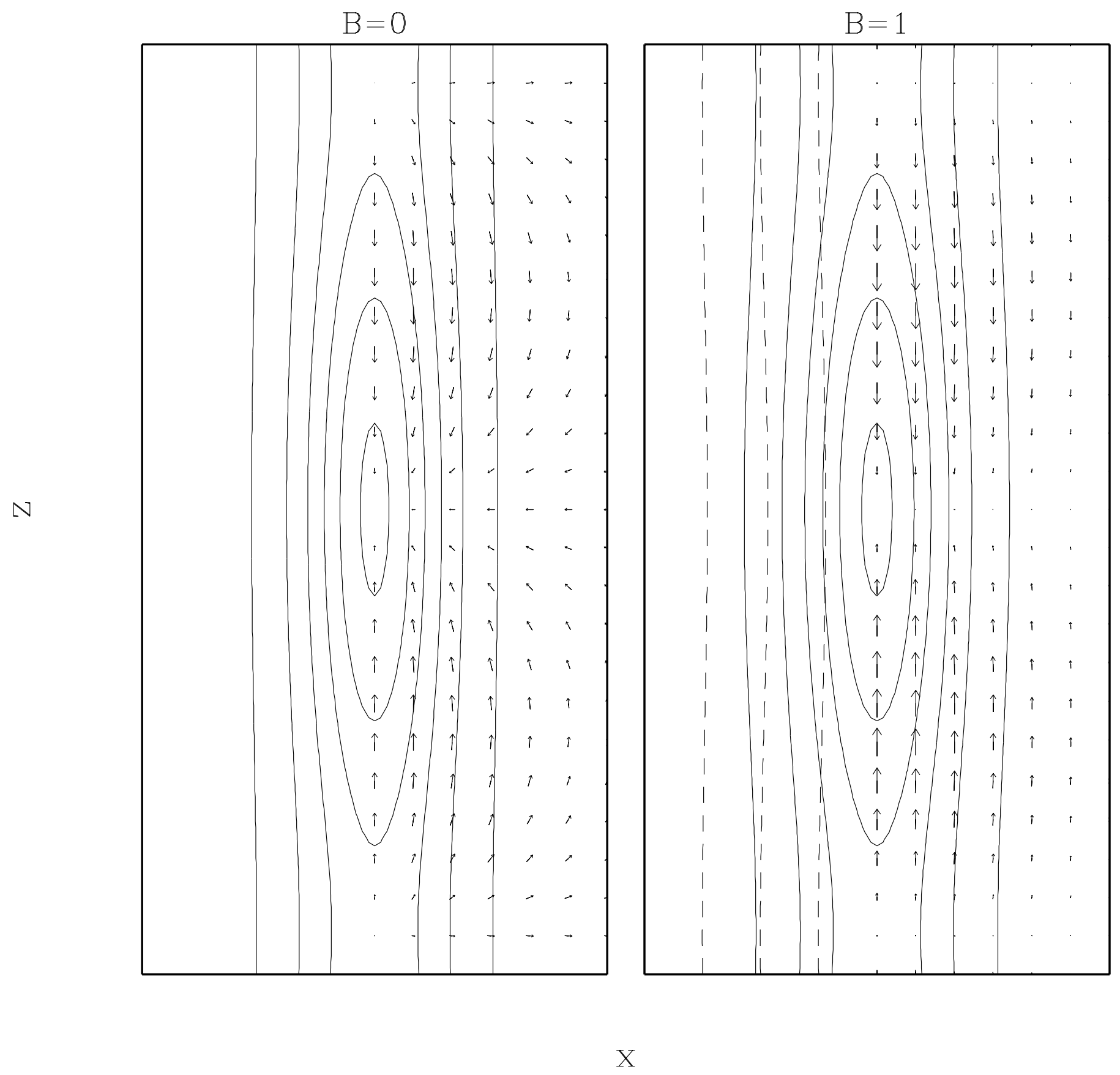

Figure 5 


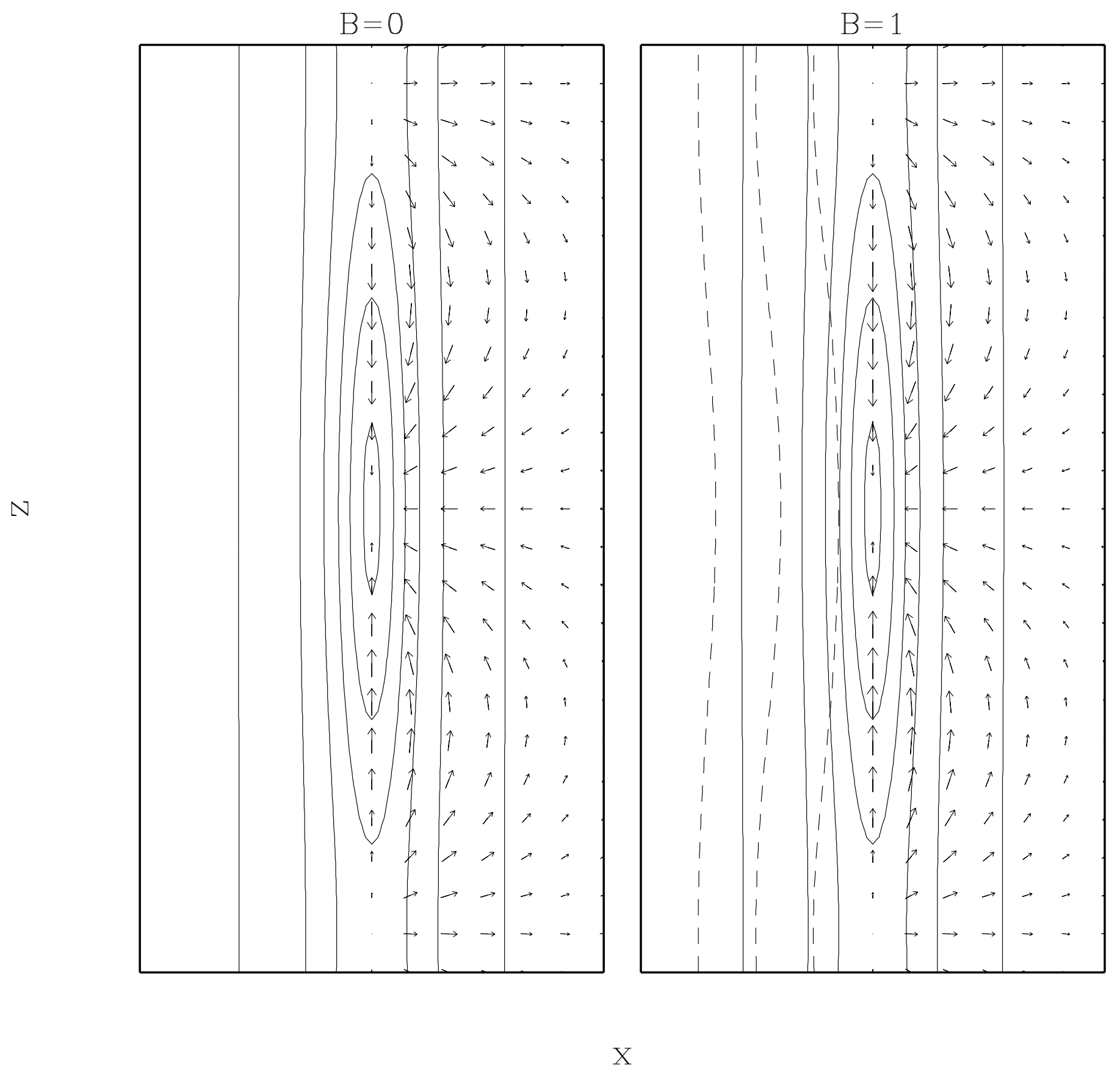

Figure 6 


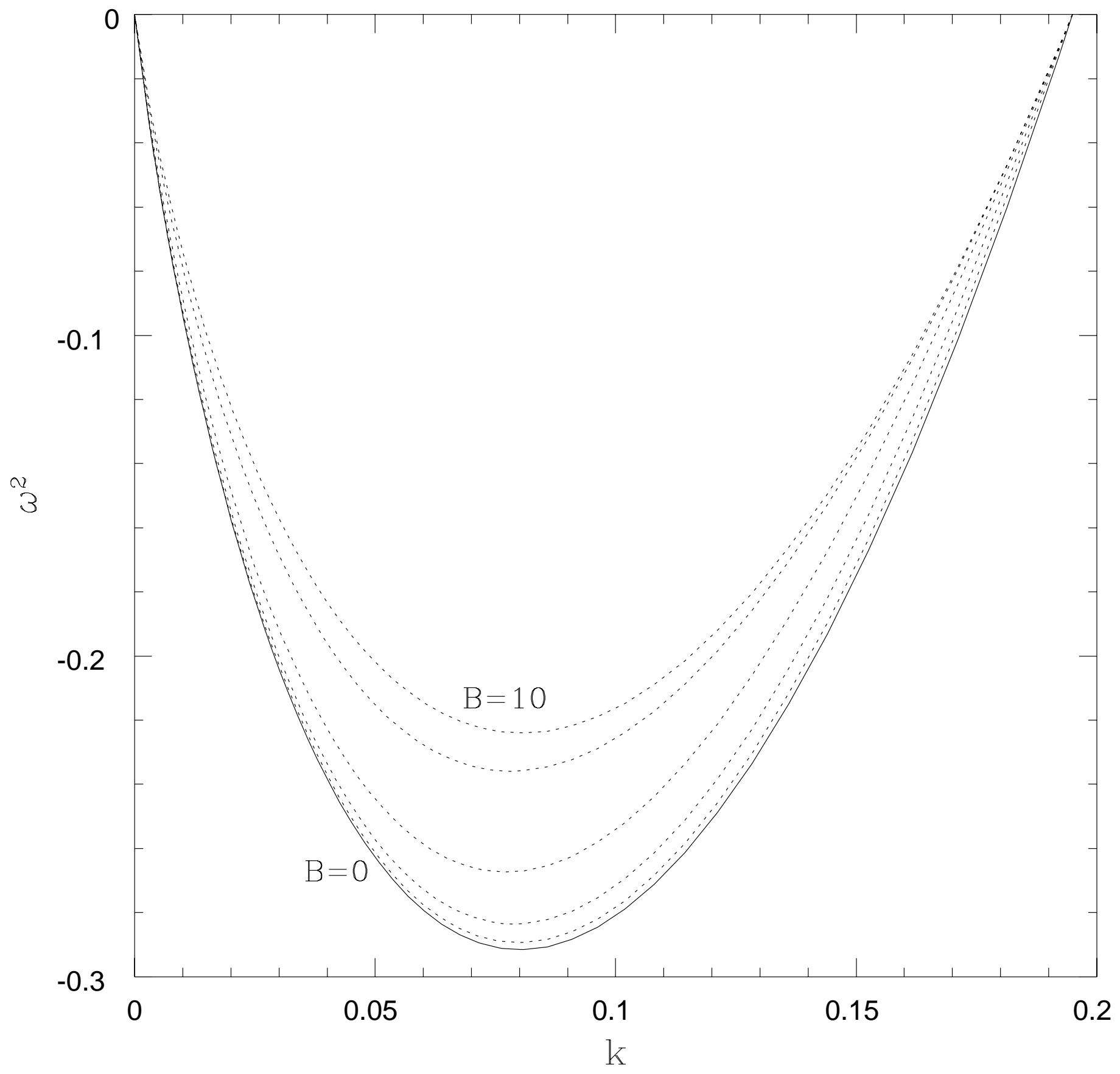

Figure 7 


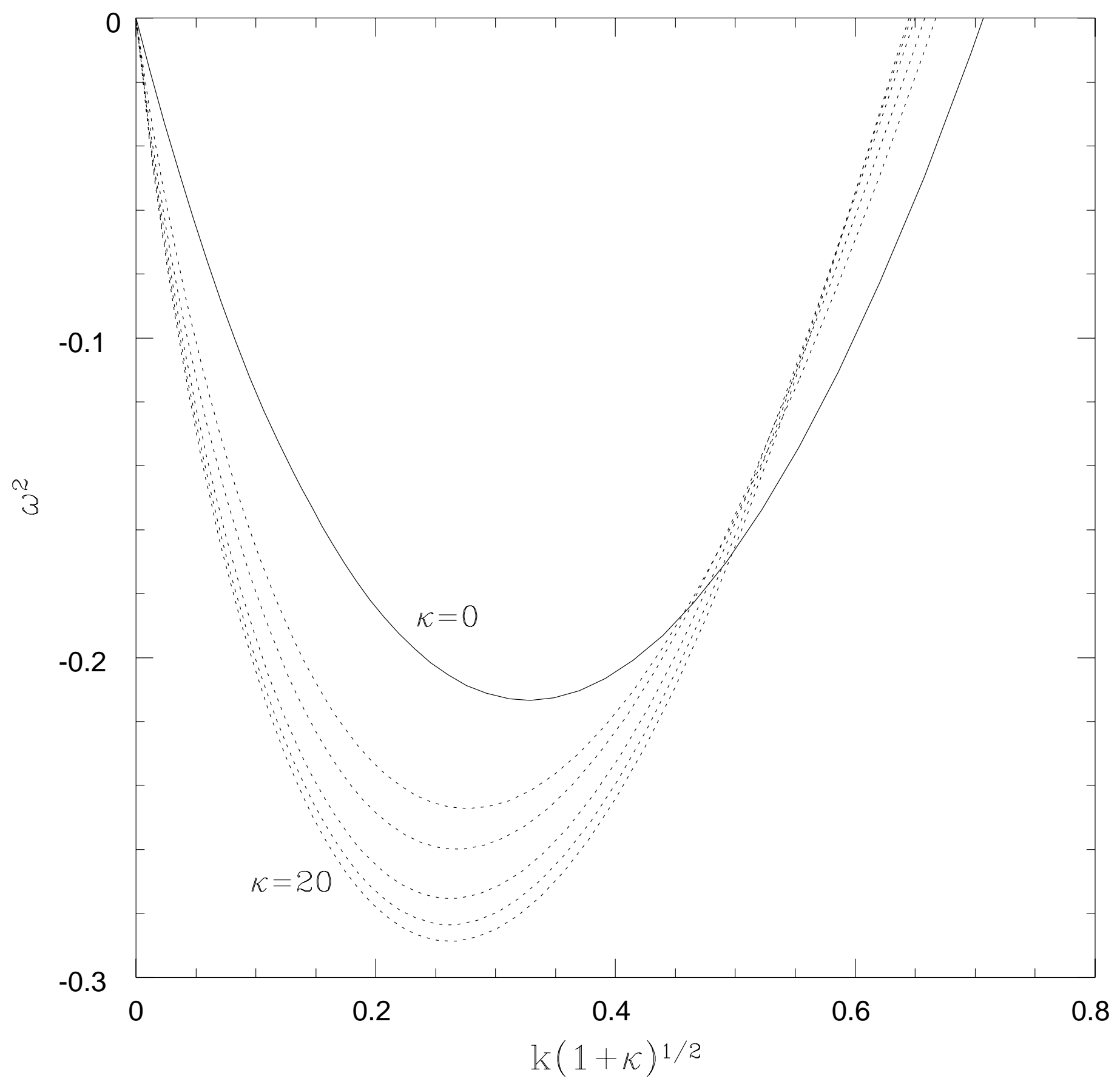

Figure 8 


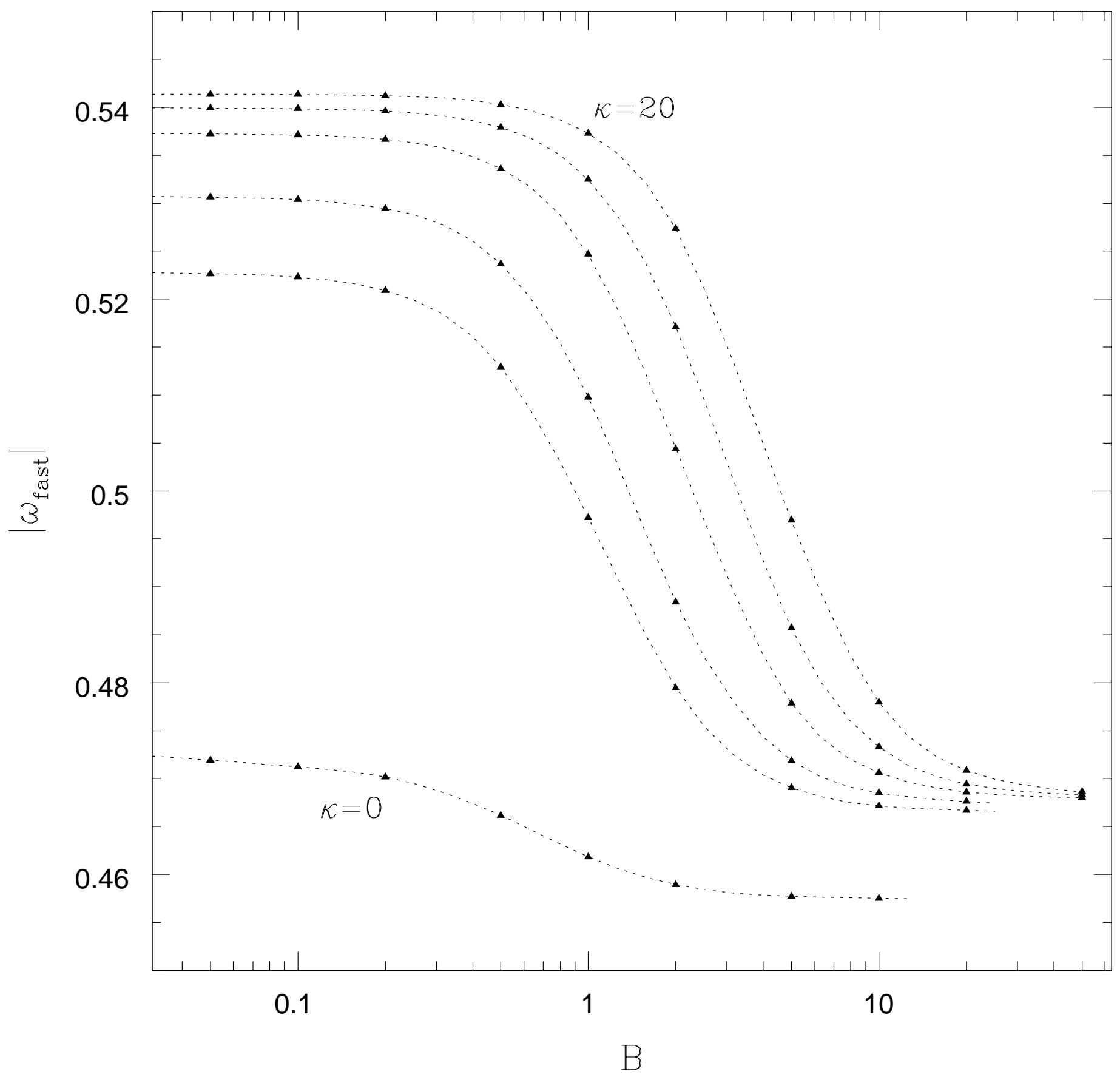

Figure 9 